\title{
Gene conversion yields novel gene combinations in paralogs of GOT1 in the copepod Tigriopus californicus
}

\author{
Christopher S Willett
}

\begin{abstract}
Background: Gene conversion of duplicated genes can slow the divergence of paralogous copies over time but can also result in other interesting evolutionary patterns. Islands of genetic divergence that persist in the face of gene conversion can point to gene regions undergoing selection for new functions. Novel combinations of genetic variation that differ greatly from the original sequence can result from the transfer of genetic variation between paralogous genes by rare gene conversion events. Genetically divergent populations of the copepod Tigriopus californicus provide an excellent model to look at the patterns of divergence among paralogs across multiple independent evolutionary lineages.

Results: In this study the evolution of a set of paralogous genes encoding putative aspartate transaminase proteins (called GOT1 here) are examined in populations of the copepod T. californicus. One pair of duplicated genes, GOT1P1 and GOT1p2, has regions of high divergence between the copies in the face of apparent on-going gene conversion. The GOT1p2 gene also has unique haplotypes in two populations that appear to have resulted from a transfer of genetic variation via inter-paralog gene conversion. A second pair of duplicated genes GOT1Sr and GOT1Sd also shows evidence of gene conversion, but this gene conversion does not appear to have maintained each as a functional copy in all populations.

Conclusions: The patterns of conservation and sequence divergence across this set of paralogous genes among populations of $T$. californicus suggest that some interesting evolutionary patterns are occurring at these loci. The results for the GOT1P1/GOT1p2 paralogs illustrate how gene conversion can factor in the creation of a mosaic pattern of regions of high divergence and low divergence. When coupled with rare gene conversion events of divergent regions, this pattern can result in the formation of novel proteins differing substantially from either original protein. The evolutionary patterns across these paralogs show how gene conversion can both constrain and facilitate diversification of genetic sequences.
\end{abstract}

Keywords: Gene duplication, Concerted evolution, Cytoplasmic GOT, Aspartate transaminase, Tigriopus californicus

\section{Background}

Gene conversion can impact the evolution of duplicated genes in a number of different ways including both impeding sequence divergence between genes and transferring variation between them [1]. Gene conversion is a common mechanism of unidirectional homologous recombination in eukaryotes that results in a cut-and-paste like copying of sequence between similar alleles that are

Correspondence: willett4@email.unc.edu

Department of Biology, University of North Carolina, Chapel Hill, NC 27599-3280, USA either at the same locus or at another locus in the same genome (reviewed in Chen et al. [2]). Concerted evolution can result from loci undergoing repeated gene conversion, which causes duplicated genes to evolve in tandem and not diverge from one another over evolutionary time. Not all duplicated genes are subject to gene conversion, in fact, surveys in mammals and fruit flies suggest that only about ten percent of paralogous copies show signs of gene conversion, and only a small fraction of the total sequence length is typically impacted [3,4].

Duplicated genes that are experiencing concerted evolution typically will go through a series of phases of differential

\section{Biomed Central}

(c) 2013 Willett; licensee BioMed Central Ltd. This is an Open Access article distributed under the terms of the Creative Commons Attribution License (http://creativecommons.org/licenses/by/2.0), which permits unrestricted use, distribution, and reproduction in any medium, provided the original work is properly cited. 
divergence. Rates of gene conversion between sequences go down as the sequences become more dissimilar. For gene duplicates undergoing some level of concerted evolution, divergence between them will not begin to increase markedly until a threshold of sequence divergence is breached (as high as 20 percent [5]). Models of this process suggest that there will typically be a long period of evolution with only low levels of divergence until a threshold level of divergence is passed at which point the rate of divergence will increase [6]. Selective divergence can counter this homogenization and lead to the establishment and maintenance of regions of higher sequence divergence in the face of gene conversion if differences in specific regions of the gene between the two duplicates are adaptive (e.g. with neofunctionalization [7]). Teshima and Innan [7] propose scanning for this specific pattern as a method of identifying such regions undergoing selection. Using this method in a study in yeast, Takuno and Innan [8] identified two sets of duplicated heat shock proteins that likely fit this model.

In addition to the role outlined above in slowing or countering adaptive divergence between duplicated genes, gene conversion can also play a role in transferring adaptive variation between duplicate genes. Under such a scenario, gene conversion acts to increase the effective population size of the duplicated genes, making selection more efficient. This transfer can spread advantageous variation and remove deleterious mutations $[9,10]$. A number of studies have shown that gene conversion between duplicate genes with some degree of initial divergence between them can result in the introduction of high levels of variation at the converted locus [11-18]. For many of these cases, this variation appears to be adaptive with a number of these genes under selection for higher haplotype diversity (e.g. MHC, attacin, and resistance genes in plants).

The copepod Tigriopus californicus has a set of unique features that makes it useful system in which to look at patterns of molecular evolution in duplicated genes. $T$. californicus exists in a series of extensively genetically divergent populations that have undergone substantial periods of independent evolution from one another. This species occurs in rocky upper intertidal pools along the Pacific coast of North America from Mexico to Alaska. Populations of this species can be highly genetically divergent from one another even over relatively short distances, with mitochondrial DNA (mtDNA) divergences greater than 20 percent between populations [19-21]. Divergence in the nuclear genome is lower but still substantial, likely reflecting a substantially higher rate of mutation for the mtDNA [22]. Even with these higher rates of mtDNA evolution, the levels of divergence among populations suggest that these populations have been evolving fairly independently of one another for long periods of time. Genomic resources are being developed for this species and now include published transcriptomes from a pair of populations, and these resources facilitate the characterization of paralogs [23].

In this paper the molecular evolution of a set of aspartate transaminase-encoding homologs is examined in populations of $T$. californicus. A putatively mitochondrially targeted homolog was previously identified from this species [24] and named after the corresponding allozyme locus (GOT2, the enzyme aspartate transaminase was formerly called glutamate-oxaloacetate transamine; EC 2.6.1.1). Five additional homologs are described in this paper that have originated from a series of gene duplication events in the evolutionary lineage leading to this species. Sequence similarity suggests that these genes are likely to be cytoplasmically targeted GOT1 proteins. Two sets of somewhat more recently duplicated pairs of genes show strong evidence of gene conversion. In this paper the differential impact of gene conversion on the evolution of these two pairs of duplicated genes is examined.

\section{Results}

\section{Identification of GOT paralogs}

Five new paralogous genes were identified from a PCRbased screen of expressed sequences from the copepod $T$. californicus that are homologous to genes encoding aspartate transaminase proteins (in addition to the previously identified GOT2 gene [24]). These genes were sequenced in four populations including three from southern California, San Diego (SD), La Jolla (LJS), and Abalone Cove $(\mathrm{AB})$, and one from central California, Santa Cruz (SCN). Four of these homologs, GOT1p1/GOT1p2 and GOT1Sd/GOT1Sr, had moderate levels of genetic divergence within pairs (Table 1) but high levels of divergence between pairs (43 percent amino acid identity). These two pairs are also highly divergent from the paralog GOT1_6a, with 41 percent and 39 percent amino acid identity between GOT1_6a and the GOT1p1 and GOT1Sr proteins respectively. The GOT1p1/GOT1p2 proteins appear to be orthologous to other arthropod cytoplasmic GOT1 proteins (58 percent amino acid identity with Drosophila melanogaster GOT1 isoform A). Phylogenetic analyses clearly place the GOT1p1/GOT1p2 paralogs with other arthropod cytoplasmic GOT1 proteins and confirm the close relationship of the GOT1Sd/GOT1Sr proteins but do not consistently resolve the relationships of these two proteins and GOT1_6a with other organisms' homologs (Additional file 1: Figure S1). These three proteins fall basal to the other GOT1 homologs from animals, but this placement in not strongly supported in either Bayesian or parsimony phylogenetic analyses.

In addition to the large amount of amino acid divergence among the more divergent GOT1 paralogs, there are also a number of structural differences at these loci. The GOT1_6a gene and the GOT1Sr genes each have 
Table 1 Fixed genetic divergence in coding regions for orthologs and paralogs of GOT1 in populations of $T$. californicus

\begin{tabular}{|c|c|c|c|c|c|c|c|c|c|c|}
\hline & \multicolumn{4}{|c|}{ Between GOT1p1/GOT1p2 paralogs } & \multicolumn{3}{|c|}{ GOT1p1 orthologs } & \multicolumn{3}{|c|}{ GOT1p2 orthologs } \\
\hline & SD p1/SD p2 & LJS p1/LJS p2 & AB p1/AB p2 & SCN p1/SCN p2 & SD p1/LJS p1 & SD p1/AB p1 & SD p1/SCN p1 & SD p2/LJS p2 & $\mathrm{SD} p 2 / \mathrm{AB} \mathrm{p} 2$ & SD p2/SCN p2 \\
\hline$k_{s}$ & 0.237 & 0.245 & 0.354 & 0.354 & 0 & 0.046 & 0.082 & 0 & 0.050 & 0.062 \\
\hline$k_{a}$ & 0.025 & 0.024 & 0.032 & 0.033 & 0 & 0.002 & 0.003 & 0 & 0.006 & 0.003 \\
\hline \multirow[t]{3}{*}{$k_{a} / k_{s}$} & 0.105 & 0.100 & 0.091 & 0.094 & - & 0.048 & 0.040 & - & 0.111 & 0.053 \\
\hline & \multicolumn{4}{|c|}{ Between GOT1Sd/GOT1Sr paralogs } & \multicolumn{3}{|c|}{ GOT1Sd orthologs } & \multicolumn{3}{|c|}{ GOT1Sr orthologs } \\
\hline & $\mathrm{SD} \mathrm{Sd} / \mathrm{SD} \mathrm{Sr}$ & LS Sd/LJS Sr & $A B S d / A B S r$ & $\mathrm{SCN} \mathrm{Sd} / \mathrm{SCN} \mathrm{Sr}$ & SD Sd/LJS Sd & SD Sd/AB Sd & $\mathrm{SD} \mathrm{Sd} / \mathrm{SCN} \mathrm{Sd}$ & SD Sr/LJS Sr & $\mathrm{SD} \mathrm{Sr} / \mathrm{AB} \mathrm{Sr}$ & $\mathrm{SD} \mathrm{Sr} / \mathrm{SCN} \mathrm{Sr}$ \\
\hline$k_{s}$ & 0.034 & 0.016 & 0.009 & 0.036 & 0.008 & 0.010 & 0.048 & 0.004 & 0.027 & 0.052 \\
\hline$k_{a}$ & 0.010 & 0.002 & 0.015 & 0.016 & 0.003 & 0.018 & 0.020 & 0.002 & 0.017 & 0.018 \\
\hline \multirow[t]{3}{*}{$k_{a} / k_{s}$} & 0.307 & 0.155 & 1.557 & 0.461 & 0.311 & 1.845 & 0.419 & 0.624 & 0.653 & 0.354 \\
\hline & \multicolumn{7}{|c|}{ GOT1_6a orthologs } & & & \\
\hline & SD 6a/LJS 6a & SD 6a/AB 6a & SD 6a/SCN 6a & LJS 6a/AB 6a & LJS 6a/SCN 6a & AB 6a/SCN 6a & & & & \\
\hline$k_{s}$ & 0.004 & 0.032 & 0.040 & 0.045 & 0.058 & 0.028 & & & & \\
\hline$k_{a}$ & 0.001 & 0.002 & 0.011 & 0.004 & 0.013 & 0.011 & & & & \\
\hline$k_{a} / k_{s}$ & 0.316 & 0.077 & 0.279 & 0.084 & 0.219 & 0.399 & & & & \\
\hline
\end{tabular}

Levels of divergence in coding region are calculated for fixed differences (excluding polymorphism) for $\mathrm{k}_{\mathrm{s}}$ (synonymous substitutions per site) and $\mathrm{k}_{\mathrm{a}}$ (non-synonymous substitutions per site) with a Jukes-Cantor correction. Raw numbers of changes and sites can be found in Additional file 2: Table S1. Note that the GOT1Sd sequences spanned only one-half of the coding region of GOT1Sr (504 bp vs 1122 bp) and that the reading frame was corrected when calculating numbers of synonymous and nonsynonymous substitutions. 
four introns in the same positions in the gene (as assessed by their position in the amino acid alignment). GOT2 also has four introns but only one of these shares a position with those of the GOT1_6a and GOT1Sr genes (the third intron). The size of this third intron varies widely from 152 bp in GOT1_6a to 3894 bp for the SCN population for GOT1Sr (the other three populations each have a $2723 \mathrm{bp}$ for this intron in the GOT1Sr gene). Interestingly, the GOT1p1/GOT1p2 genes lack introns completely. The transcript for each of these genes is between 1257 bp for GOT1Sr and 1532 bp for GOT1_6a, while the coding regions are all close to 1224 bp (with GOT1_6a being $1233 \mathrm{bp}$ ). We did not obtain sequence corresponding to the first $618 \mathrm{bp}$ of the coding region for the GOT1Sd gene, but the sequenced portion is consistent with the presence of the final three introns. For the $A B$ and LJS populations the second and third introns respectively have polymorphisms that would alter the predicted splice sequences for the GOT1Sd gene.

Five of these six GOT genes can be found in the published transcriptome dataset derived from the SD and SCN populations of $T$. californicus [23] with only the GOT1Sd gene missing. These data also give some hints as to the relative expression levels of these genes. Total read numbers per gene are somewhat low overall in this 454 dataset but the highest counts were found for the GOT2 gene with 247 reads and the GOT1p1/GOT1p2 genes with 154 reads summed over both copies. Examination of the proportion of reads from the diagnostic regions of the GOT1p1/GOT1p2 genes suggests that the expression of the GOT1p1 gene is about 6-fold higher than that of the GOT1p2 gene. The GOT1_6a and GOT1Sr genes had fewer than 10 reads each suggesting that they are expressed at a much lower level. Consistent with its absence from the transcriptomes, our lab found no expression of the GOT1Sd gene using qualitative RTPCR assays in the San Diego (SD) population, but we did find expression of both the GOT1Sr and GOT1p1/ GOT1p2 paralogs (Willett CS, unpublished data). Sequences of mRNA obtained from individual copepods from these experiments were identical to haplotypes obtained via direct sequencing from the coding regions.

\section{Divergence in GOT1 paralogs and gene conversion}

The duplication events that produced the GOT1p1 and GOT1p2 paralogs and the GOT1Sd and GOT1Sr paralogs appear to have occurred in the Tigriopus lineage prior to the split of the four populations examined in this study. This can be seen for the GOT1p1 and GOT1p2 paralogs in the higher levels of divergence between paralogs within a population as compared to the divergence between presumed orthologs between populations (Table 1) and from phylogenetic analyses (Figure 1A). In both of the GOT1p1 and GOT1p2 paralogs there are more synonymous substitutions than nonsynonymous substitutions between orthologous copies across populations and this is reflected in the low values of $\mathrm{K}_{\mathrm{a}} / \mathrm{K}_{\mathrm{s}}$ (with GOT1p1 showing a higher degree of conservation). Comparisons across paralogous copies within populations show the same pattern of relatively low $\mathrm{K}_{\mathrm{a}} / \mathrm{K}_{\mathrm{s}}$ values (Additional file 2: Table $\mathrm{S} 1$ ). The number of nonsynonymous substitutions is higher across populations for the GOT1Sd and GOT1Sr paralogs with correspondingly higher $\mathrm{K}_{\mathrm{a}} / \mathrm{K}_{\mathrm{s}}$ ratios (with some exceeding one; Table 1). The GOT1Sd and GOT1Sr paralogs have not diverged substantially in the sequenced coding regions, but the third intron has diverged to the degree that much of it cannot be aligned between the paralogs (it also differs significantly in size-761 bp for GOT1Sd and $2723 \mathrm{bp}$ for GOT1Sr in the SD population).

It appears that a history of past and on-going gene conversion events has left a strong impression on the patterns of genetic variation within and among paralogs of GOT1 in T. californicus. Using the program GENECONV [25] a series of inter- and intra paralog conversion events are evident (Figures 2 and 3; Additional file 3: Table S2). If we count non-overlapping predicted gene conversion events as a minimum number of gene conversion events, there have been at least two inter-paralog and one intraparalog gene conversion events between GOT1p1 and GOT1p2 genes for SD and LJS populations (with the intraparalog events occurring between alleles of GOT1p2). A minimum of two inter-paralog gene conversion events are also predicted for the $\mathrm{AB}$ population and one for the $\mathrm{SCN}$ population with no intra-paralog events for either population (predicted conversion events are listed in Additional file 3: Table S2). For the GOT1Sd and GOT1Sr paralogs there are a minimum of two inter-paralog gene conversion events predicted for the SD, LJS, and $\mathrm{AB}$ populations and one for the SCN population. For this pair, intra-paralog gene conversion events are limited to the GOT1Sd paralog, and there are at a minimum two of these for the SCN population and one for the SD and LJS populations. Also, for the GOT1Sd and GOT1Sr paralogs, the inter-paralog gene conversion events are largely restricted to the exons (with the third intron too divergent in sequence to align over much of its length as mentioned previously). The predicted intra-paralog gene conversion events for GOT1Sd are all predicted to occur in this same intron (Additional file 3: Table S2). In contrast, for the GOT1p1 and GOT1p2 paralogs, there are no introns in the sequenced region of the gene, and the inter-paralog gene conversion events overlap primarily with the regions of low genetic divergence between paralogs. Comparisons of GOT1 homologs from a range of arthropods and two vertebrates suggest that conserved amino acid regions are scattered across the protein and not centered only in the regions with evidence for gene conversion events (Additional file 4: Figure S2). 


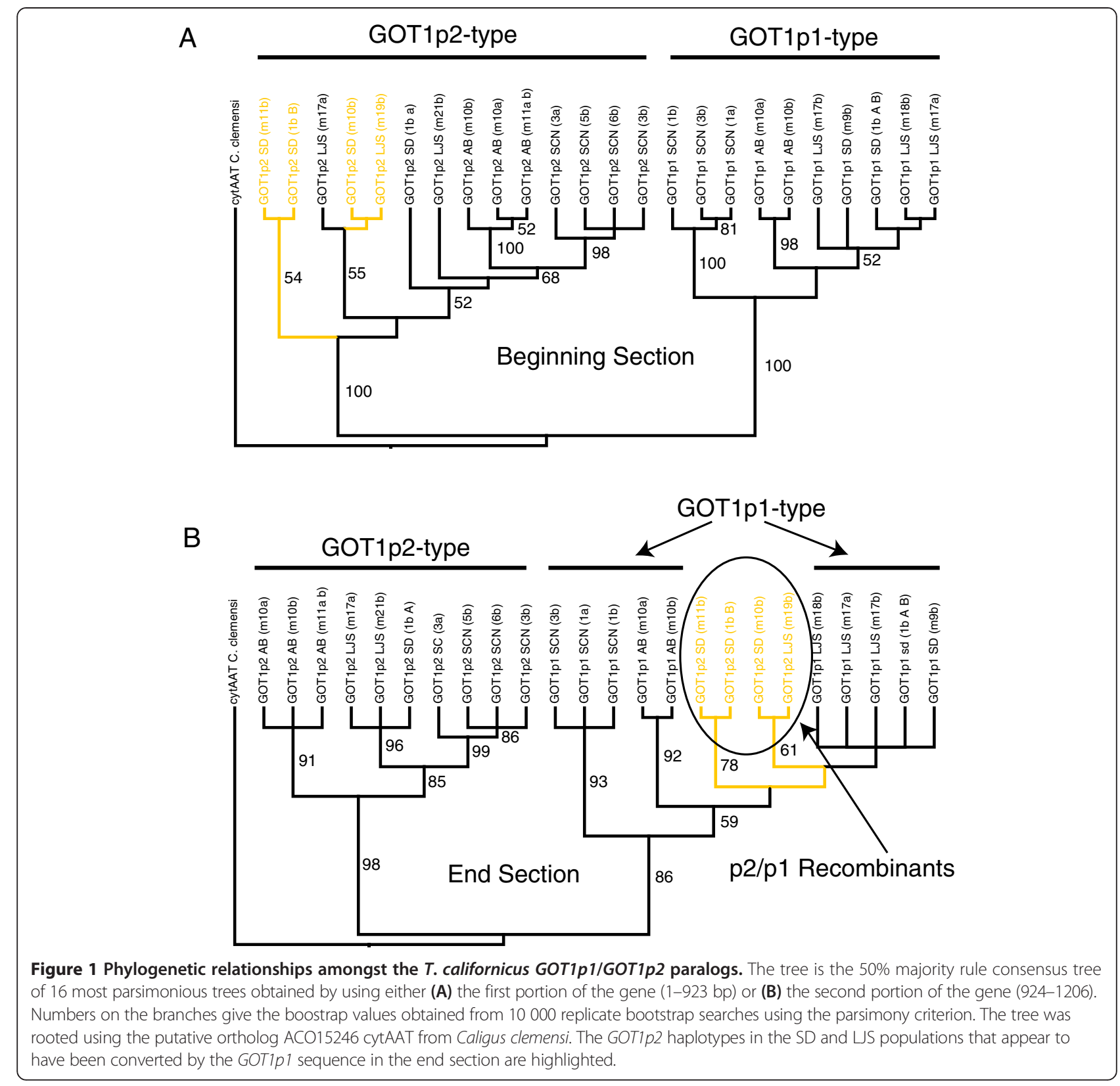

Even for the regions with no genetic divergence between GOT1p1 and GOT1p 2 paralogs from the same population, there is still some divergence among populations for each ortholog (Figure 4).

\section{Polymorphism capture via gene conversion}

The SD and LJS GOT1p1 and GOT1p2 paralogs stand out for their elevated levels of polymorphism in comparison to other genes in these populations (Table 2; $[22,26])$, and it appears that this pattern could stem from inter-paralog gene conversion events introducing variation. For these genes levels of synonymous polymorphism are nearly an order of magnitude higher than levels from other genes in these T. californicus populations, which had an average $\pi$ value of 0.003 for SD and 0.006 for LJS across a set of eight nuclear-encoded genes [22,26]. The GOT1p 2 genes in the SD and LJS populations also have a large number of nonsynonymous polymorphisms for the GOT1p2 paralog in addition to synonymous polymorphisms. For the peak of divergence at position 930 (Figure 2) alleles can differ by as many as eight nonsynonymous polymorphisms and fourteen synonymous polymorphisms. It appears that many of these polymorphisms are the result of gene conversion introducing variation from the GOT1p1 copy to the GOT1p2 copy as can be seen by examining patterns of divergence between alleles (Figure 4). 


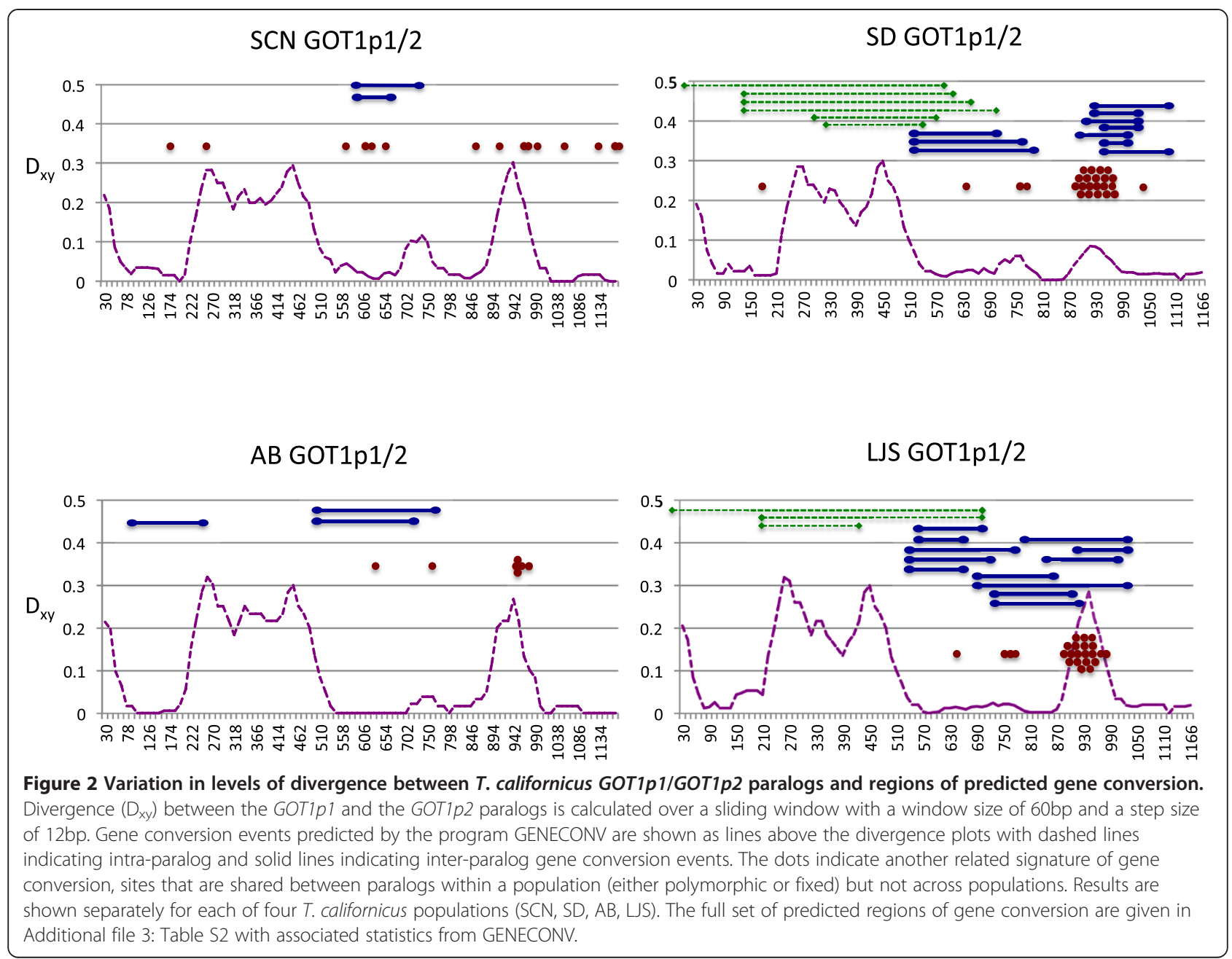

Phylogenetic analyses of each end of the gene also confirm this pattern of differential history for these SD and LJS GOT1p2 haplotypes (Figure 1). Inspection of all of the sequences also suggests a number of other candidate SNPs that are likely to result from such inter-paralog events in these two populations and in the other two populations as well (dots in Figure 2).

Although there is evidence for inter-paralog gene conversion for the GOT1Sd and GOT1Sr paralogs as well, it does not appear to have been substantial enough to result in both copies retaining their open reading frames in all haplotypes. For the SD, LJS, and particularly SCN populations there are fixed and polymorphic indels in exons in GOT1Sd that should disrupt the reading frame and result in greatly truncated mRNAs (Table 2 ). In the $\mathrm{AB}$ population there appears to be a premature stop codon in the GOT1Sr that is polymorphic in this population. For both the GOT1Sr and GOT1Sd paralogs elevated $k_{\mathrm{a}} / \mathrm{k}_{\mathrm{s}}$ ratios are seen for some comparisons further suggesting reduced functional constraint (Table 1). For the GOT1p2 gene one haplotype in the SD population also had an insertion that would disrupt the reading frame suggesting that non-functional alleles can also be found at this locus. A one bp deletion was found in the coding region for a single haplotype in GOT1_6a in the SD population as well. Only for the GOT1p1 gene copy were no such truncating or frameshift polymorphisms found in any of this set of four populations of T. californicus for these five GOT1 homologs.

\section{Discussion}

I have identified a set of homologous genes from T. californicus that appear to encode aspartate transaminase proteins and these genes display a number of interesting patterns of inter-locus gene conversion. In discussing these results, first, I will discuss the potential deeper level relationships among these duplicates within and between species and then, second, I will look at the interesting patterns of gene conversion in two pairs of more closely related duplicates.

The cytosolic GOT1 proteins have undergone a number of gene duplication events in copepods and in the $T$. 


\section{$D_{x y}$ GOT1Sd/r}

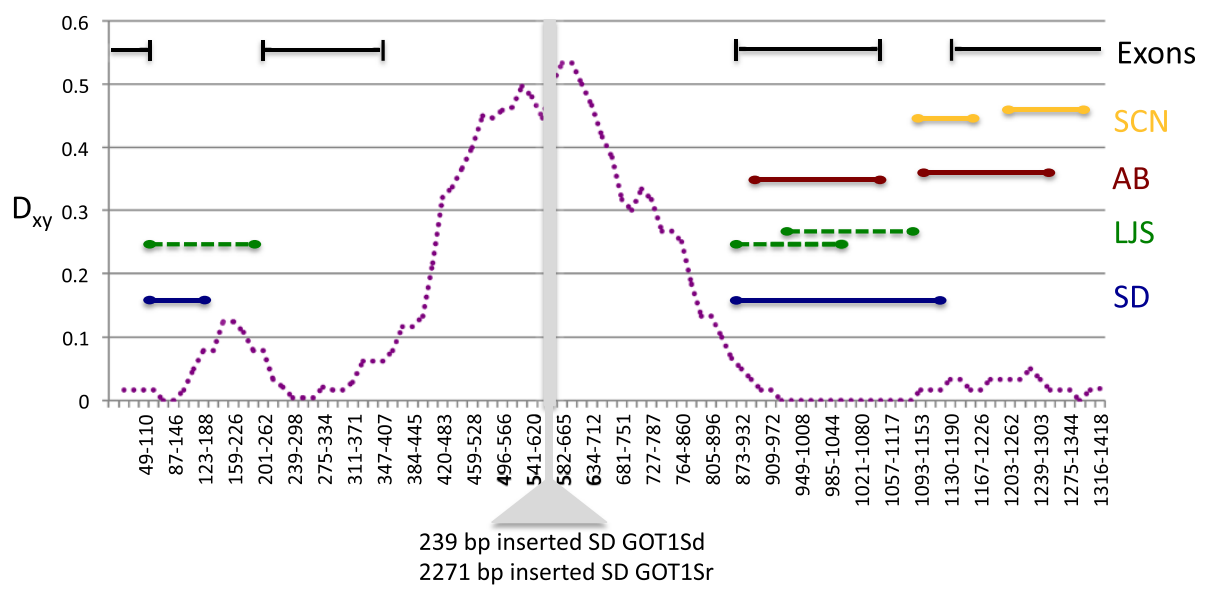

Figure 3 Levels of divergence between GOT1Sd/GOT1Sr paralogs from T. californicus with regions of predicted gene conversion. Divergence $\left(D_{x y}\right)$ is calculated across all four populations between the two paralogs of GOT1S with a sliding window of size $50 \mathrm{bp}$ and $10 \mathrm{bp}$ steps. Patterns of divergence were very similar across populations with the slight exception of the SCN population comparison that showed a small peak (0.15) of divergence centered over the 985-1044 window. Only inter-paralog conversion events are given for these two paralogs with population identity shown on the righthand side of the figure. Exonic regions are indicated at the top of the figure. Note that the central intron varies in size between GOT1Sd and GOT1Sr and cannot be aligned. The full set of predicted regions of gene conversion are given in Additional file 3: Table $\$ 2$ with associated statistics from GENECONV.

californicus lineage. The GOT1p1/GOT1p2 paralogs cluster phylogenetically with cytosolic GOT1 proteins in other species of arthropods and are their most likely orthologs. The relationships of the other three GOT1 paralogs to other GOT1 proteins are not resolved with the exception of a weakly supported relationship to putative GOT1 paralogs in two other distantly related copepod species (Caligus clemensi and Lepeoophtheirus salmonis). The lack of deeply divergent GOT1 paralogs in other sequenced metazoan genomes suggests that the duplication events producing the GOT1_6a and GOT1Sd/GOT1Sr paralogs may have occurred within copepods and were not the result of an ancient metazoan duplication event. Other examples of older duplicates of aspartate transaminases in animals are restricted to individual clades such as mammals as can be seen in panther gene family trees http://www.pantherdb.org/ for aspartate aminotransferases [27]. If the duplications did occur within copepods, perhaps relatively high levels of amino acid divergence in these paralogs are obscuring their relationship to the other GOT1 proteins. Regardless of the deeper level relationships, it is clear that the duplications that have resulted in the production of the GOT1Sd/GOT1Sr and GOT1p1/GOT1p2 gene pairs occurred more recently than these deeper splits. Most likely these splits occurred in the common ancestor of these four populations of T. californicus given the presence of each copy in each population.

The GOT1p1 paralog is the most conserved of the five paralogs with no evidence for segregating non-functional alleles (Table 2) and it has the highest levels of constraint as measured by $k_{\mathrm{a}} / \mathrm{k}_{\mathrm{s}}$ values (Table 1 ). The higher expression level of the GOT1p1 copy, coupled with potential matches between predicted amino acid differences and allozyme allele differences among populations together suggest that the GOT1p1 paralog could be the same locus as the GOT1 allozyme used previously to examine genetic variation among $T$. californicus populations $[19,28,29]$ and may be the primary cytosolic aspartate transaminase protein in this species. The GOT1p2 paralog has slightly lower levels of constraint than the GOT1p1 paralog and has one haplotype that contains a frameshift polymorphism in this sample of sequences from the SD population. Of the five paralogs, the GOT1Sd gene is behaving the most like a pseudogene. It does not appear to be expressed at detectable levels and has a series of frameshift substitutions in each of the populations that disrupt the reading frame (with the exception of the $A B$ population).

Turning now to the patterns of gene conversion in the more recently duplicated pairs of paralogs, GOT1Sd/ GOT1Sr and GOT1p1/GOT1p2, it is clear that there has been gene conversion in the past within each pair. There is no evidence of gene conversion between the more divergent paralogs, e.g. between GOT1Sr and GOT1_6a. There are numerous likely gene conversion tracks resulting from both inter- and intra-locus events between pairs for both of these sets of paralogs (Figures 1 and 2; Additional file 3: Table S2). For the GOT1Sd/GOT1Sr pair the interparalog gene conversion events are largely restricted to 

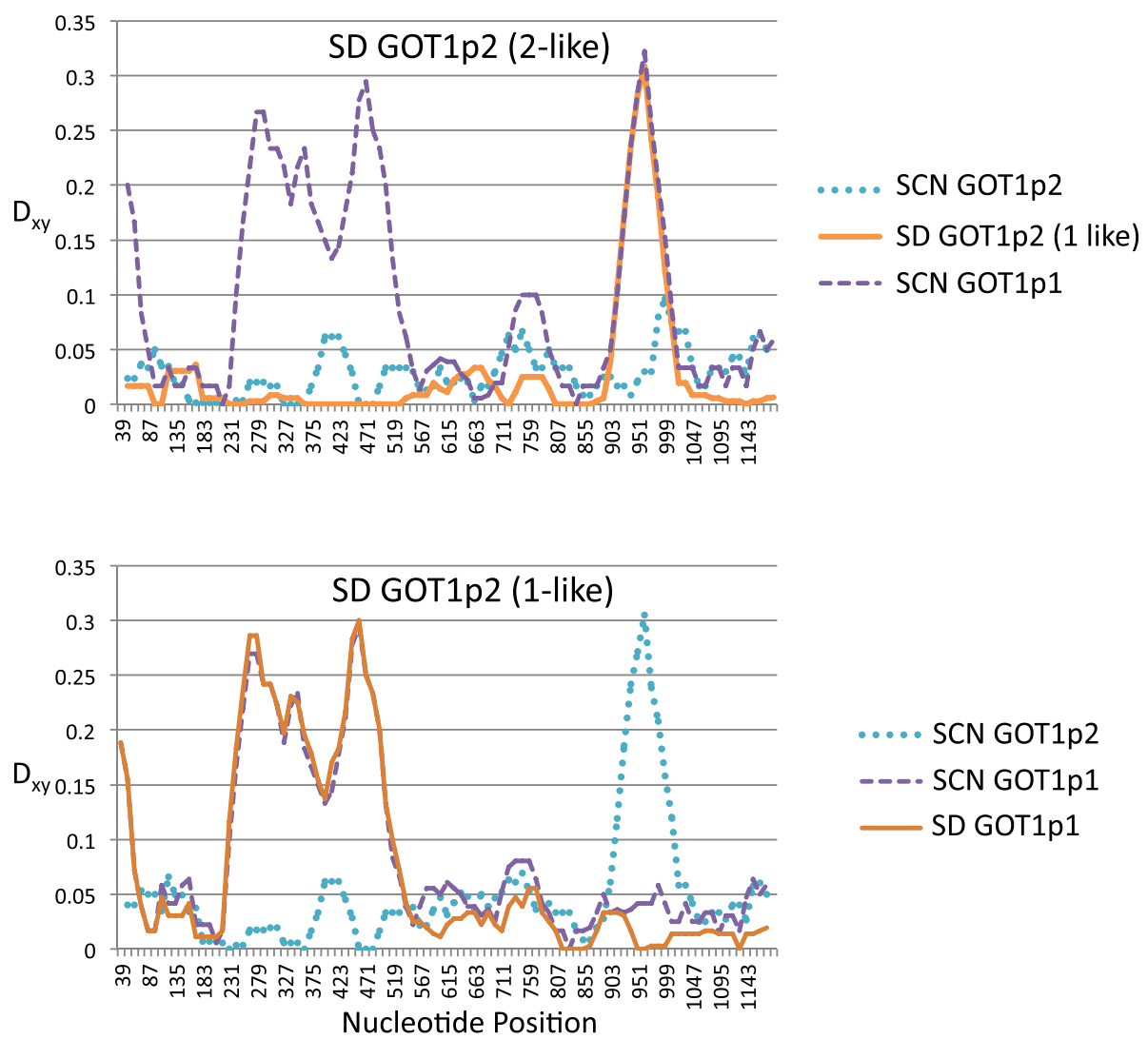

Figure 4 Apparent transfer of variation via inter-paralog gene conversion for GOT1p1 and GOT1p2. The transfer of variation from GOT1p1 to GOT1p2 for certain alleles is evident in the patterns of divergence across each gene in comparisons of SCN and SD paralogs. In the top graph comparisons are done between the SD GOT1p2 (2-like) paralog and the SD GOT1p2 (1-like), and SCN paralogs. In the bottom graph comparisons are made between the GOT1p2 (1-like) paralog and the SD GOT1p1 paralog, and SCN paralogs. The GOT1p2 (1-like) alleles appear to have been converted by the GOT1p1 copy for the peak near position 951 because they are divergent from the GOT1p2 (and 2-like) alleles but similar to the GOTp1 copies. In contrast, the GOT1p2 (2-like) alleles have no large peaks of divergence with the GOT1p2 copies from the SCN population (similar patterns are seen for comparisons with the AB and LJS paralogs).

the exonic sequences with a large intron becoming largely un-alignable between paralogs. The GOT1Sd gene appears to be evolving as a pseudogene in several populations as discussed above despite evidence for inter-locus gene conversion events with the largely intact GOT1Sr gene. Apparently these gene conversion events are not happening frequently enough to maintain the open reading frame of this GOT1Sd copy in all populations. In contrast to the GOT1Sd/GOT1Sr pair of genes, there are no introns in the coding sequences of the GOT1p1/GOT1p2 paralogs and the regions of elevated divergence between the two paralogs are therefore located within the single exon. Close physical proximity in the genome can facilitate interlocus gene conversion [30] and in fact, the GOT1p1/GOT1p2 paralogs are tightly linked (and are also located on the same chromosome as GOT2; Willett CS, unpublished data). The allozyme loci GOT1 and GOT2 were previously shown to be linked [31], lending further credence to the idea that the GOT1p1 and/or
GOT1p2 loci might encode the allozyme marker GOT1 that has been previously characterized in this species.

Both pairs of paralogs GOT1Sd/GOT1Sr and GOT1p1/ GOT1p2 show islands of genetic divergence amid regions of higher similarity but the evolutionary explanation for this pattern may differ between the two sets of duplicates. For the GOT1Sd/GOT1Sr pair the divergence is restricted to the introns and may be a result of the accumulation of substitutions that can terminate inter-paralog gene conversion in those stretches of the gene. Divergence in sequence similarity that lowers the level of gene conversion could accumulate either via the gradual accumulation of single-base differences or more rapidly by larger changes such as large indels [32,33]. The GOT1Sd/GOT1Sr paralogs have both very large size differences and low sequence similarity in the intron so that either mode of divergence could have contributed to the absence of gene conversion in these regions. Even small regions of clustered sequence divergence (with multiple substitutions 
Table 2 Levels of polymorphism in GOT paralogs in T. californicus

\begin{tabular}{|c|c|c|c|c|c|c|c|c|c|c|}
\hline Pop. & Paralog & \# Hap. & $\pi_{\text {syn }}$ & $S_{\text {syn }}$ & $\pi_{\text {nonsyn }}$ & $S_{\text {nonsyn }}$ & $\pi_{\text {syn+nc }}$ & $S_{\text {syn+nc }}$ & Taj. D & Indels in coding region \\
\hline \multirow[t]{5}{*}{ SD } & GOT1p1 & 6 & 0.06674 & 3 & 0 & 0 & NA & NA & 1.09 & \\
\hline & GOT1p2 & 10 & 0.04501 & 34 & $0.00675^{*}$ & 15 & 0.04359 & 34 & 0.21 & 70 bp insert (one haplotype) \\
\hline & GOT1Sr & 4 & 0 & 0 & 0 & 0 & 0.00076 & 5 & 0.372 & \\
\hline & GOT1Sd & 10 & 0 & 0 & $0.00538^{*}$ & 5 & 0.00466 & 12 & 1.144 & $1 \mathrm{bp}$ fixed \\
\hline & GOT1_6a & 10 & 0.00742 & 4 & 0.00119 & 2 & 0.0057 & 4 & 1.06 & 1bp poly. \\
\hline \multirow[t]{5}{*}{ LJS } & GOT1p1 & 6 & 0.02401 & 13 & 0.00195 & 4 & NA & NA & 0.74 & \\
\hline & GOT1p2 & 10 & 0.02296 & 29 & 0.00495 & 15 & 0.02223 & 29 & -1.48 & \\
\hline & GOT1Sr & 4 & 0.00186 & 1 & 0.00136 & 5 & 0.0008 & 5 & -0.446 & \\
\hline & GOT1Sd & 8 & 0.0163 & 6 & $0.00494^{*}$ & 5 & 0.00977 & 23 & 0.23 & 1 bp poly. \\
\hline & GOT1_6a & 10 & 0.00211 & 1 & 0.00044 & 1 & 0.00092 & 0 & 0.12 & \\
\hline \multirow[t]{5}{*}{$A B$} & GOT1p1 & 6 & 0.00111 & 1 & 0.0004 & 1 & NA & NA & -1.13 & \\
\hline & GOT1p2 & 6 & 0.00412 & 3 & 0.00087 & 1 & 0.00399 & 3 & 0.56 & \\
\hline & GOT1Sr & 4 & 0.00479 & 2 & $0.0023^{*}$ & 3 & 0.00102 & 4 & 1.06 & (stop codon poly.) \\
\hline & GOT1Sd & 4 & 0 & 0 & 0.00287 & 2 & 0.00045 & 1 & -0.754 & \\
\hline & GOT1_6a & 10 & 0.00214 & 1 & 0.00043 & 1 & 0.00304 & 4 & 1.5 & \\
\hline \multirow[t]{5}{*}{$\mathrm{SCN}$} & GOT1p1 & 6 & 0.01356 & 8 & 0 & 0 & NA & NA & 0.27 & \\
\hline & GOT1p2 & 10 & 0.01774 & 14 & 0.00155 & 4 & 0.01747 & 14 & -0.05 & \\
\hline & GOT1Sr & 2 & 0 & 0 & 0 & 0 & 0.00122 & 6 & NA & \\
\hline & GOT1Sd & 9 & 0.036 & 8 & $0.0123^{*}$ & 9 & 0.00632 & 9 & 0.672 & 4bp poly., $1 \mathrm{bp}$ poly., 4bp fixed \\
\hline & GOT1_6a & 8 & 0.016 & 11 & 0.0023 & 5 & 0.0165 & 14 & -0.21 & \\
\hline
\end{tabular}

\# Hap. indicates the number of haplotypes sequenced for each gene. S indicates the number of segregating sites for each type of polymorphism (syn-synonymous, non-syn-nonsynonymous, syn+nc-noncoding and synonymous). $\pi$ gives the average pairwise sequence divergence for each of the same three catergories of sites. Taj. D is the value of Tajima's D. Poly. indicates that site is polymorphic.

*Note calculations of non-synonymous polymorphism correct reading frame caused by indels in coding region for comparison to other sequences.

or indels) can dramatically reduce the rate of gene conversion for a region of a gene $[34,35]$. The net result of this divergence for the GOT1Sd/GOT1Sr paralogs is that interlocus gene conversion is not likely to occur in this intronic region of the gene and these regions are free to accumulate further differences.

In contrast for the GOT1p1/GOT1p2 paralogs the regions of genetic divergence occur in the exons and there are no fixed indels in these regions that could disrupt interlocus gene conversion. Teshima and Innan [7] have suggested that such regions of differentiation in the face of on-going gene conversion can be a signal that selection is maintaining divergence in the paralogs (i.e. the paralogs have begun the process of neofunctionalization). Under such a model the width of the divergent region should extend less than the average length of a gene conversion tract from the selected site or sites. A number of duplicated genes show such islands of divergence that are associated with clear functional differences in the resulting proteins (e.g. RH factor and opsin proteins [36]). Other duplicated genes in yeast and Drosophila show a similar pattern consistent with selection but lack evidence for functional differences [8,37]. For the GOT1p1/GOT1p2 paralogs, one potential neutral explanation for this pattern could posit that gene conversion initiation is lower in these regions and that these regions have accumulated enough differentiation to begin to suppress gene conversion. An argument against this limited initiation idea is that intralocus gene conversion is common in the region of sequence differentiation between these two paralogs in the first half of the gene. This observation suggests that sequence factors are not completely suppressing the initiation of gene conversion events in the divergent regions of the gene. Other factors that could also suppress interlocus gene conversion such as indel differences are also absent. The loss of fixed divergences between paralogs for one of these islands of genetic divergence in the SD and LJS populations in the second half of the gene (discussed further below) also argues that gene conversion is still possible for these regions. Although these results are suggestive of a selective explanation, further study attempting to identify functional differences between the GOT1p1/GOT1p2 paralogs is needed to confirm or reject this hypothesis.

A region of high polymorphism and lowered divergence between a set of alleles in the GOT1p1/GOT1p2 paralogs in the SD and LJS populations is likely to have been created by inter-paralog gene conversion. The patterns of variation and phylogenetic evidence (Figure 1 
and Figure 4) are consistent with one-way transfers of variation from each population's GOT1p1 locus to the GOT1p2 locus. One-way exchange like this is consistent with other studies where gene conversion shows biased directionality $[2,38]$. The net result of this directional gene conversion is to transfer variants from one paralog to the other. In this case this transfer is limited to the second half of the gene resulting in haplotypes that are a chimera of the GOT1p1 and the GOT1p2 paralogs and this transfer also results in an increase in the levels of polymorphism in this region of the gene. The chimeric protein that results is substantially altered from that produced by other GOTp 2 alleles, differing by 8 amino acids, while still differing from GOTp1 by 20 amino acid in the first half of the gene.

It is possible that gene conversion events that result in greatly augmented polymorphism in gene duplicates are effectively neutral, but in a number of other cases they appear to be under selection, often occurring in genes undergoing selection for diversification [11-17,39]. For the GOT1p1 and GOT1p2 genes there is not a clear signal of diversifying selection in comparisons of orthologous copies across populations with $\mathrm{K}_{\mathrm{a}} / \mathrm{K}_{\mathrm{s}}$ values much lower than one (Table 2). Without any further functional information it is difficult to say whether the gene conversion events that resulted in greatly increased diversity in the GOT1p2 gene in the SD and LJS population are adaptive in nature. Clearly this process has generated a large amount of novel variation at this locus both in DNA and protein sequence.

\section{Conclusions}

The two sets of duplicate genes of GOT1 illustrate different patterns of evolution with ongoing gene conversion among duplicated copies. The set of GOT1Sd/GOT1Sr genes appear to be in the process of diverging with gradually decreasing gene conversion given that one copy does not maintain its open reading frame and does not appear to be expressed. The central intron in this gene is already quite divergent. In contrast for the GOT1p1/GOT1p 2 pair, gene conversion is maintaining much higher similarity in some regions of the gene but other exonic portions are substantially diverged. The combination of these islands of genetic divergence between paralogs with rare gene conversion events has the ability to construct radically different haplotypes from the combination of variation in both paralogs (as has happened in the SD and LJS GOT1p2 gene). Further work on the function of these two duplicates could help to determine whether there are likely to be adaptive differences between these copies.

\section{Methods}

\section{Isolation and sequencing of GOT1 homologs}

The putative GOT1 homologs were uncovered from T. californicus using an analogous strategy to that used to obtain the GOT2 homolog in this species [24]. Briefly, a cDNA library was screened for putative homologs using a PCR-RACE procedure with primers designed to match conserved regions of GOT proteins from a range of species. Five homologs of GOT1 were eventually identified using this screen after cloning and sequencing the products to separate the more closely related paralogs. Initial work was done for the San Diego population in southern California (SD, $32.7457^{\circ} \mathrm{N}, 117.2550^{\circ} \mathrm{W}$, San Diego County, CA). Three other sites were used to examine the evolution of these GOT1 paralogs, two more in southern California, La Jolla (LJS, $32.8434^{\circ} \mathrm{N}, 117.2808^{\circ} \mathrm{W}$, San Diego County, $\mathrm{CA})$, and Abalone Cove $\left(\mathrm{AB}, 33.7377^{\circ} \mathrm{N}, 118.3753^{\circ} \mathrm{W}\right.$, Los Angeles County, CA), and one site in central California, Santa Cruz (SCN, $36.9495^{\circ} \mathrm{N}, 122.0470^{\circ} \mathrm{W}$, Santa Cruz County, CA). These sites were selected because they capture a number of divergent lineages of T. californicus and have been used extensively in other studies of sequence evolution in this species $[22,24,26]$.

To obtain the sequences of each of these five GOT1 paralogs, DNA from single copepods was obtained using a proteinase-K cell-lysis method [40]. Table 3 lists the primers that were used in PCR reactions that can specifically amplify each paralogous sequence under the specified set of conditions. PCR products were directly sequenced using capillary sequencing. Between two and

Table 3 Primer sequences and amplification conditions for GOT1 paralogs from $T$. californicus

\begin{tabular}{lllll}
\hline Gene Region & Forward Primer (5' to $\mathbf{3}^{\prime}$ ) & Reverse Primer (5' to $\mathbf{3}^{\prime}$ ) & Size (bp) & Ann. Temp. \\
\hline GOT1p1 & AGAAGTTGTCATTCATTCTTCATC & CTTATTGACGGCCTCATTGATGGA & 1243 & $58^{\circ} \mathrm{C}$ \\
GOT1p2 & ATATCCGTGCCCAAAGCCTAC & CTTATTGACGGCCTCATTGATGGA & 1249 & $58^{\circ} \mathrm{C}$ \\
GOT1Sr (5'end) & AGTATCCCAACATGTCTGCCTTCG & GCAATATAGATAATGAATAACGACACT & $\sim 1700^{1}$ & $63^{\circ} \mathrm{C}^{2}$ \\
GOT1Sr (middle) & GAGTGTCGTTATTCATTATCTATATTGC & TCCTCAACCAGGTTTAGAGTCA & $1751 \mathrm{SD}, 1722 \mathrm{AB}, 2855 \mathrm{SCN}$ & $63^{\circ} \mathrm{C}$ \\
GOT1Sr (3'end) & CAATTGACTCTAAAACCTGGTTGA & GGTTATTCTTGAATTGTTGTGCTTCGT & $\sim 1400 \mathrm{bp}$ & $63^{\circ} \mathrm{C}$ \\
GOT1Sd & CAGGAACAATGGAAGATCATAGCCA & GGTTATTCTTGAATTGTTGTGCTTCGT & $\sim 1700 \mathrm{bp}$ & $\sim 1600 \mathrm{bp}$ \\
GOT1_6A & ATGGCCACAACCAAGTTATTG & TGGTCTTTGATGGGGCCTTCGTTCG & $55^{\circ} \mathrm{C}$ \\
\hline
\end{tabular}

${ }^{1}$ There are small size variations due to indels in these fragments.

${ }^{2}$ For the amplifications of the GOT1Sr gene fragments the Phusion kit (NEB) was used to amplify products. 
ten haplotypes were sequenced for each gene from each of the four populations (the numbers of sequenced haplotypes are given in Table 2). To verify the sequence of the expressed mRNA for these paralogs, RNA from single copepods was isolated using the TRI reagent RNA isolation procedure (Sigma Chemical, Saint Louis, MO). After making cDNA from these preparations, the mRNA sequence was obtained from individual copepods from each of the four populations listed above for the two GOT1p1/2 paralogs and the GOT1Sr gene (there did not appear to be any product from the GOT1Sd gene in these populations).

\section{Sequence analyses}

All sequences were aligned and edited using Sequencer v4.8 software (Genecodes, Ann Arbor, Michigan). The program DNAsp v.5 [41] was used to perform the polymorphism and divergence analyses for each gene. In addition to calculations of polymorphism and divergence (including analyses over sliding windows), Tajima's D test [42] was also implemented. The program GENECONV (version 1.81a http://www.math.wustl.edu/ sawyer/geneconv/) was used to identify regions of the paralogous genes that have sequence patterns consistent with gene conversion [25]. Gene conversion events were identified both within and between paralogs within a single population by setting up the group structure within the file and allowing only gene conversion events within populations. The protein variability server (http://imed.med.ucm.es/PVS/) was used to look at patterns of amino acid conservation across GOT1 proteins of arthropods [43]. Conservation was measured by looking at the diversity of amino acids at each site using the Shannon entropy $\mathrm{H}$ value.

Phylogenetic trees were constructed using both parsimony and Bayesian analyses with amino acid sequence data and only with parsimony for DNA sequence data from within Tigriopus. The program PAUP"v4b10-×86 [44] was used for the parsimony reconstructions of relationships among GOT1p1/GOT1p2 haplotypes. Heuristic searches were done with 100 random starting trees using either the first $923 \mathrm{bp}$ of the sequence or the last $282 \mathrm{bp}$ in separate analyses. A similar search approach was used for analyses of the divergent sets of GOT amino acid sequences for parsimony analyses. A variety of search conditions using Bayesian analyses and the program MrBayes v3.1.2 [45] were also performed on these protein alignments but did not provide strong support for unresolved relationships in the parsimony analyses.

\section{Availability of supporting data}

Sequences are available in Genbank with the accession numbers [KF135593 to KF135616]. The data sets (sequence alignments) supporting the results of this article are available in the Dryad repository http://dx.doi.org/10.5061/ dryad.8r6jp.

\section{Additional files}

Additional file 1: Figure S1. Most parsimonious tree for relationships among GOT paralogs.

Additional file 2: Table S1. Genetic divergence among orthologs and paralogs of GOT1 in T. californicus. Numbers of fixed substitutions and sites are for comparisons of GOT1 homologs.

Additional file 3: Table S2. List of potential sites of gene conversion. Results are obtained from the program GENE_CONV.

Additional file 4: Figure S2. Plot of conservation of GOT1 proteins from arthropods with divergent regions of T. californicus GOT1p1/GOT1p2 highlighted.

\section{Competing interests}

The author declares no competing interests, financial or otherwise, regarding this manuscript.

\section{Acknowledgements}

E. Burch, A. Craven, H. Kunduru collected much of the data for this paper and helped with the analyses. T. Lima provided helpful comments on the manuscript. This work supported by the National Science Foundation (grant DEB-0821003 and IOS-1155325). Two anonymous reviewers provided useful comments as well on a previous draft.

Received: 26 March 2013 Accepted: 8 July 2013

Published: 12 July 2013

\section{References}

1. Innan H: Population genetic models of duplicated genes. Genetica 2009, 137(1):19-37.

2. Chen JM, Cooper DN, Chuzhanova N, Ferec C, Patrinos GP: Gene conversion: mechanisms, evolution and human disease. Nat Rev Genet 2007, 8(10):762-775.

3. McGrath CL, Casola C, Hahn MW: Minimal effect of ectopic gene conversion among recent duplicates in four mammalian genomes. Genetics 2009, 182(2):615-622.

4. Casola C, Ganote CL, Hahn MW: Nonallelic gene conversion in the genus Drosophila. Genetics 2010, 185(1):95-103.

5. Mansai SP, Kado T, Innan H: The rate and tract length of gene conversion between duplicated genes. Genes 2011, 2(4):313-331.

6. Teshima KM, Innan H: The effect of gene conversion on the divergence between duplicated genes. Genetics 2004, 166(3):1553-1560.

7. Teshima KM, Innan H: Neofunctionalization of Duplicated Genes Under the Pressure of Gene Conversion. Genetics 2008, 178(3):1385-1398.

8. Takuno S, Innan $\mathrm{H}$ : Selection to maintain paralogous amino acid differences under the pressure of gene conversion in the heat-shock protein genes in yeast. Mol Biol Evol 2009, 26(12):2655-2659.

9. Mano $\mathrm{S}$, Innan $\mathrm{H}$ : The evolutionary rate of duplicated genes under concerted evolution. Genetics 2008, 180(1):493-505.

10. Fawcett J, Innan H: Neutral and non-neutral evolution of duplicated genes with gene conversion. Genes 2011, 2(4):191-209.

11. Gorski J, Mach B: Polymorphism of human la antigens: gene conversion between two DR $\beta$ loci results in new HLA-D/DR specificity. Nature 1986 322(6074):67-70.

12. Winderickx J, Battisti L, Hibiya Y, Motulsky AG, Deeb SS: Haplotype diversity in the human red and green opsin genes: Evidence for frequent sequence exchange in exon 3. Hum Mol Genet 1993, 2(9):1413-1421.

13. Parniske M, Hammond-Kosack KE, Golstein C, Thomas CM, Jones DA, Harrison K, Wulff BBH, Jones JDG: Novel disease resistance specificities result from sequence exchange between tandemly repeasted genes at the Cf-4/9 locus of tomato. Cell 1997, 91(6):821-832.

14. Charlesworth D, Bartolome C, Schierup MH, Mable BK: Haplotype structure of the stigmatic self-incompatibility gene in natural populations of Arabidopsis lyrata. Mol Biol Evol 2003, 20(11):1741-1753. 
15. Lazzaro BP, Clark AG: Evidence for recurrent paralogous gene conversion and exceptional allelic divergence in the Attacin genes of Drosophila melanogaster. Genetics 2001, 159(2):659-671.

16. Nielsen KM, Kasper J, Choi M, Bedford T, Kristiansen K, Wirth DF, Volkman SK, Lozovsky ER, Hartl DL: Gene conversion as a source of nucleotide diversity in Plasmodium falciparum. Mol Biol Evol 2003, 20(5):726-734.

17. Storz JF, Baze M, Waite JL, Hoffmann FG, Opazo JC, Hayes JP: Complex signatures of selection and gene conversion in the duplicated globin genes of house mice. Genetics 2007, 177(1):481-500.

18. Rane HS, Smith JM, Bergthorsson U, Katju V: Gene conversion and DNA sequence polymorphism in the sex-determination gene fog-2 and its paralog ftr-1 in Caenorhabditis elegans. Mol Biol Evol 2010, 27(7):1561-1569.

19. Burton RS: Intraspecific phylogeography across the Point Conception biogeographic boundary. Evolution 1998, 52(3):734-745.

20. Edmands S: Phylogeography of the intertidal copepod Tigriopus californicus reveals substantially reduced population differentiation at northern latitudes. Mol Ecol 2001, 10(7):1743-1750.

21. Willett CS, Ladner JT: Investigations of fine-scale phylogeography in Tigriopus californicus reveal historical patterns of population divergence. BMC Evol Biol 2009, 9(1):139.

22. Willett CS: Quantifying the elevation of mitochondrial DNA evolutionary substitution rates over nuclear rates in the intertidal copepod Tigriopus californicus. J Mol Evol 2012, 74(5-6):310-318.

23. Barreto FS, Moy GW, Burton RS: Interpopulation patterns of divergence and selection across the transcriptome of the copepod Tigriopus californicus. Mol Ecol 2011, 20(3):560-572.

24. Willett CS: Complex deleterious interactions associated with malic enzyme may contribute to reproductive isolation in the copepod Tigriopus californicus. PLoS One 2011, 6(6):e21177.

25. Sawyer SA: GENECONV: A computer package for the statistical detection of gene conversion. 1999. Distributed by the author, available at http://www. math.wustl.edu/ sawyer/geneconv/.

26. Willett CS: Evolution of interacting proteins in the mitochondrial electron transport system in a marine copepod. Mol Biol Evol 2004, 21(3):443-453.

27. Thomas PD, Kejariwal A, Campbell MJ, Mi HY, Diemer K, Guo N, Ladunga I, Ulitsky-Lazareva B, Muruganujan A, Rabkin S, et al: PANTHER: a browsable database of gene products organized by biological function, using curated protein family and subfamily classification. Nucleic Acids Res 2003, 31(1):334-341.

28. Burton RS, Feldman MW: Population genetics of Tigriopus californicus. II. Differentiation among neighboring populations. Evolution 1981, 35(6):1192-1205

29. Burton RS: Genetic evidence for long term persistence of marine invertebrate populations in an ephemeral environment. Evolution 1997, 51(3):993-998.

30. Lichten M, Haber JE: Position effects in ectopic and allelic mitotic recombination in Saccharomyces cerevisiae. Genetics 1989, 123(2):261-268.

31. Dill MM, Burton RS: Genetics of mitochondrial glutamate-oxaloacetate transaminase (GOT-2) in Tigriopus californicus. Biochem Genet 1984 22(3-4):339-347

32. Walsh JB: Sequence-dependent gene conversion: can duplicated genes diverge fast enough to escape conversion. Genetics 1987, 117(3):543-557.

33. Chen $\mathrm{WL}$, Jinks-Robertson $\mathrm{S}$ : The role of the mismatch repair machinery in regulating mitotic and meiotic recombination between diverged sequences in yeast. Genetics 1999, 151(4):1299-1313.

34. Godwin AR, Liskay RM: The effects of insertions on mammalian intrachromosomal recombination. Genetics 1994, 136(2):607-617.

35. Lukacsovich T, Waldman AS: Suppression of intrachromosomal gene conversion in mammalian cells by small degrees of sequence divergence. Genetics 1999, 151(4):1559-1568.

36. Innan $\mathrm{H}$ : A two-locus gene conversion model with selection and its application to the human RHCE and RHD genes. P Natl Acad Sci USA 2003, 100(15):8793-8798

37. Osada N, Innan H: Duplication and gene conversion in the Drosophila melanogaster genome. PLoS Genet 2008, 4(12):e1000305.

38. Bosch E, Hurles ME, Navarro A, Jobling MA: Dynamics of a human interparalog gene conversion hotspot. Genome Res 2004, 14(5):835-844.

39. Ohta T: Role of Diversifying Selection and Gene Conversion in Evolution of Major Histocompatibility Complex Loci. P Natl Acad Sci USA 1991 88(15):6716-6720
40. Hoelzel AR, Green A: Analysis of population-level variation by sequencing PCR-amplified DNA. In Practical Approach Series: Molecular Genetic Analysis Of Populations. Edited by Hoelzel AR. New York, New York: Oxford University Press; 1992:159-187.

41. Librado P, Rozas J: DnaSP v5: a software for comprehensive analysis of DNA polymorphism data. Bioinformatics 2009, 25(11):1451-1452.

42. Tajima F: Statistical methods for testing the neutral mutation hypothesis by DNA polymorphism. Genetics 1989, 123(3):585-595.

43. Garcia-Boronat M, Diez-Rivero CM, Reinherz EL, Reche PA: PVS: a web server for protein sequence variability analysis tuned to facilitate conserved epitope discovery. Nucleic Acids Res 2008, 36:W35-W41.

44. Swofford DL: PAUP*. phylogenetic analysis using parsimony ( ${ }^{*}$ and other methods). In., 4 edn. Sunderland, MA: Sinauer Associates; 1993.

45. Ronquist F, Huelsenbeck JP: MrBayes 3: Bayesian phylogenetic inference under mixed models. Bioinformatics 2003, 19(12):1572-1574.

doi:10.1186/1471-2148-13-148

Cite this article as: Willett: Gene conversion yields novel gene combinations in paralogs of GOT1 in the copepod Tigriopus californicus. BMC Evolutionary Biology 2013 13:148.

\section{Submit your next manuscript to BioMed Central and take full advantage of:}

- Convenient online submission

- Thorough peer review

- No space constraints or color figure charges

- Immediate publication on acceptance

- Inclusion in PubMed, CAS, Scopus and Google Scholar

- Research which is freely available for redistribution 